\title{
Structure-Based Identification of Inhibitory Fragments Targeting the p300/CBP-Associated Factor Bromodomain
}

\author{
Apirat Chaikuad, ${ }^{\dagger}$ Steffen Lang, ${ }^{\perp}$ Paul E. Brennan, ${ }^{\dagger}$ Claudia Temperini, ${ }^{\S}$ Oleg Fedorov, ${ }^{\dagger}$ \\ Johan Hollander," Ruta Nachane, $"$ Chris Abell, ${ }^{\perp}$ Susanne Müller, ${ }^{\dagger}$ Gregg Siegal, $\|$ and Stefan Knapp*, ${ }^{*},+$ \\ ${ }^{\dagger}$ Nuffield Department of Clinical Medicine, Structural Genomics Consortium and Target Discovery Institute, University of Oxford, \\ Old Road Campus Research Building, Roosevelt Drive, Oxford, OX3 7DQ, U.K. \\ ${ }^{*}$ Institute for Pharmaceutical Chemistry, Johann Wolfgang Goethe-University and Buchmann Institute for Molecular Life Sciences, \\ Max-von-Laue-Strasse 9, D-60438 Frankfurt am Main, Germany \\ ${ }^{\S}$ Leiden Institute of Chemistry, Leiden University, Einsteinweg 55, 2333 CC Leiden, Netherlands \\ ${ }$ ZoBio, Einsteinweg 55, 2333 CC Leiden, Netherlands \\ ${ }^{\perp}$ Department of Chemistry, University of Cambridge, Lensfield Road, Cambridge CB2 1EW, U.K.
}

\section{Supporting Information}

ABSTRACT: The P300/CBP-associated factor plays a central role in retroviral infection and cancer development, and the $\mathrm{C}$-terminal bromodomain provides an opportunity for selective targeting. Here, we report several new classes of acetyl-lysine mimetic ligands ranging from $\mathrm{mM}$ to low micromolar affinity that were identified using fragment screening approaches. The binding modes of the most attractive fragments were determined using high resolution crystal structures providing chemical starting points and structural models for the development of potent and selective PCAF inhibitors.

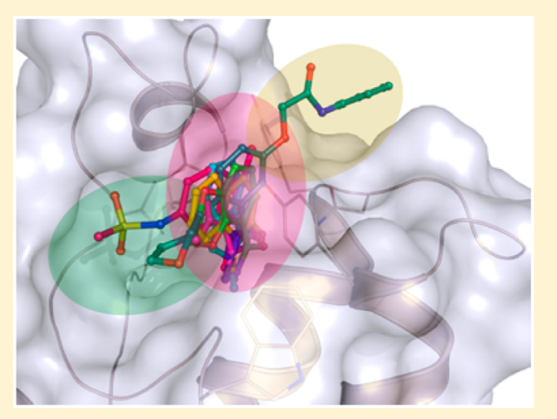

\section{INTRODUCTION}

Bromodomains are epigenetic readers that specifically recognize acetyl-lysine (Kac) marks on proteins. Targeting this protein interaction module with small molecules has recently emerged as a potential therapeutic strategy for the treatment of several diseases including cancer and inflammation. ${ }^{1,2}$ To date, most inhibitor development efforts have been focused on the BET family of bromodomain proteins for which several inhibitors have now entered clinical testing. Bromodomains have good predicted druggability, ${ }^{3}$ and selective chemical tool compounds have been developed even for less attractive binding sites that possess open or highly charged acetyl-lysine binding pockets such as $\mathrm{BAZ2}^{4,5}$ and ATAD2. ${ }^{6}$ In addition, potent inhibitors have been developed for highly druggable bromodomains present in $\mathrm{BRPF},{ }^{7,8} \mathrm{CBP},{ }^{9}$ and BRD9. ${ }^{10}$ Interestingly, several recent inhibitor development projects have highlighted the success of fragment-based approaches identifying inhibitors, in particular for poorly druggable bromodomains. $5,6,8,11,12$

P300/CBP-associated factor (PCAF, also known as histone acetyltransferase KAT2B) is a multidomain protein that harbors an acetyltransferase (HAT) and E3 ubiquitin ligase domains as well as a C-terminal bromodomain that may associate with the HATs P300 and CBP. ${ }^{13-15}$ While the roles of the acetyltransferase and the E3 ubiquitin ligase activities have been shown to be required for cell proliferation and apoptosis, ${ }^{14-18}$ little is known about the regulatory function of the PCAF bromodomain in cellular processes. Selective inhibitors, so-called chemical probes, would therefore be interesting reagents to unravel the functions of the PCAF bromodomain and to assess its therapeutic potential as a targeting site for drug development.

An important role of the PCAF acetyl-lysine recognition module has already been demonstrated for the replication of AIDS viruses. The PCAF bromodomain targets the HIV TAT protein acetylated at $\mathrm{K} 50$, an essential association that activates HIV-1 transcription and promotes the integrated proviral replication. ${ }^{19,20}$ Development of PCAF bromo-domain inhibitors has therefore been proposed as a potential strategy for the treatment of AIDS, ${ }^{19,20}$ and this strategy has been confirmed by early N1-aryl-propane-1,3-diamine-based compounds that have been shown to prevent PCAF/TAT association. ${ }^{21}$

This compound class has recently been followed up by the development of a series of 2-(3-aminopropylamino) pyridine 1oxide derivatives that however did not lead to improvement of the micromolar potency of current PCAF bromodomain inhibitors. $^{22}$ This prompted us to identify more diverse chemical starting points using fragment screening by thermal shifts assays and NMR as well as structure-based approaches.

Special Issue: Epigenetics

Received: November 4, 2015

Published: January 5, 2016 


\section{RESULTS AND DISCUSSION}

To extend structural knowledge from our previous apo crystal structure, ${ }^{23}$ we initially determined the crystal structure of the PCAF-acetyl-lysine complex to provide insights into acetyllysine recognition. The binding mode of the acetyl-lysine in PCAF strongly resembled that observed in other bromodomains with the Kac carbonyl forming two canonical hydrogen bonds to a conserved $\mathrm{N} 803$ and a conserved water molecule. This water is part of a conserved intricate network of structural waters located at the bottom of the pocket that bridge interaction with the ZA loop Y760 and the backbone carbonyl of E750 (Figure 1a). Despite sharing the conserved
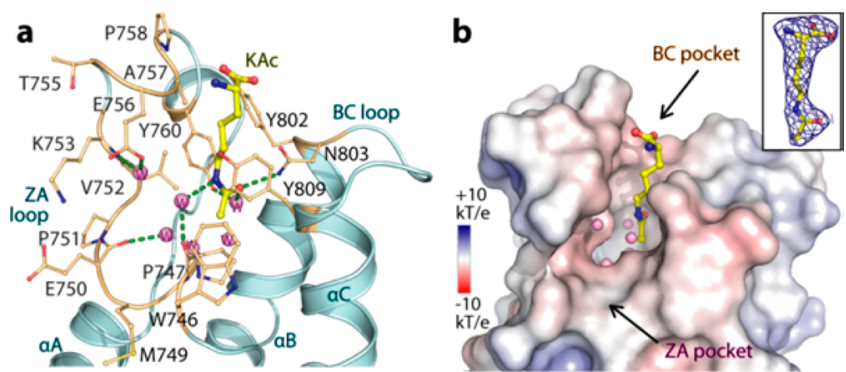

Figure 1. Structure of PCAF-Kac complex (PDB code 5fe0). (a) Detailed interactions between the bound acetyl-lysine (Kac, shown in yellow stick) and the PCAF bromodomain. Water molecules are shown as pink spheres. (b) Electrostatic surface representation reveals a tight central Kac pocket that is extended by wide cavities lined by the $\mathrm{ZA}$ and $\mathrm{BC}$ loop regions. Inset shown $\left|2 F_{\mathrm{o}}\right|-\left|F_{c}\right|$ omitted electron density map for the bound Kac.

interactions, the Kac binding groove in PCAF was fenced on one side by the bulky residue Y809, resulting in a tight and restricted pocket (Figure $1 \mathrm{~b}$ ). Large aromatic residues in this position are not common for human bromodomains, present in less than a third of proteins in the family. ${ }^{23}$

Another interesting structural feature was the extension of the binding pocket by a shelf created by the ZA loop harboring a mixture of hydrophobic and polar surface residues (Figure 1b). The wide ZA-loop pocket poses a challenge for inhibitor development. However, recently the development of Baz2 inhibitors demonstrated that such large binding pockets can be efficiently occupied for instance by aromatic ring systems of the BAZ2-ICR probe that are aligned by intramolecular aromatic $\pi$ stacking. ${ }^{4}$

The low affinities of the previously developed compounds prompted us to search for other Kac mimetics that would be compatible with the constraint nature of the central PCAF pocket. We employed different techniques to screen two fragment libraries. In one set of experiments, we used a thermal stability shift assay to screen a small fragment set at $1 \mathrm{mM}$ and identified several fragments that resulted in positive melting temperature $\left(T_{\mathrm{m}}\right)$ shifts in the range of $\sim 1-2{ }^{\circ} \mathrm{C}$ (Figure 2). These hits were chemically diverse and contained different potential Kac mimetic scaffolds. The highest $T_{\mathrm{m}}$ shifts were recorded for 1-methylquinolin-2(1H)-one) (1, BR004), $1 H$ indole-5-carboxamide (2, BR005), 2-methylisoindolin-1-one (3, BR013), 4-methoxybenzo[d]isoxazol-3-amine (4, MB360), 2,3dihydrobenzo $[b][1,4]$ dioxine-5-carboxamide (5, MB364), and (4-(1,2,3-thiadiazol-4-yl)phenyl)methanamine (7, MB093), most of which comprised known Kac mimetic scaffolds. In parallel, we used target immobilized NMR screening (TINS) to screen a diverse collection of approximately 950 commercially a

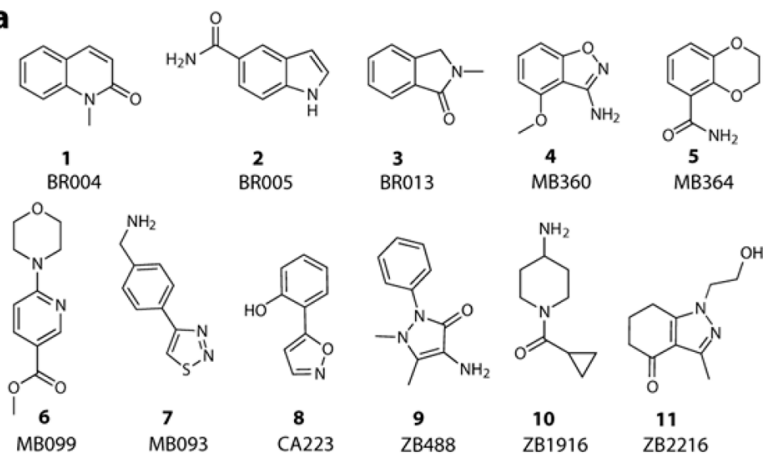

b

\begin{tabular}{c|c|c|c|c|c|c|c|c|c|c|c|}
\hline fragment & $\mathbf{1}$ & $\mathbf{2}$ & $\mathbf{3}$ & $\mathbf{4}$ & $\mathbf{5}$ & $\mathbf{6}$ & $\mathbf{7}$ & $\mathbf{8}$ & $\mathbf{9}$ & $\mathbf{1 0}$ & $\mathbf{1 1}$ \\
\hline $\begin{array}{c}\Delta \mathrm{Tm}\left({ }^{\circ} \mathrm{C}\right) \\
\text { at } 1 \mathrm{mM} \\
( \pm \mathrm{SEM})\end{array}$ & $\begin{array}{c}2.0 \\
(0.5)\end{array}$ & $\begin{array}{c}2.0 \\
(0.4)\end{array}$ & $\begin{array}{c}1.5 \\
(0.5)\end{array}$ & $\begin{array}{c}0.8 \\
(0.4)\end{array}$ & $\begin{array}{c}0.8 \\
(0.3)\end{array}$ & $\begin{array}{c}0.7 \\
(0.3)\end{array}$ & $\begin{array}{c}1.0 \\
(0.4)\end{array}$ & $\begin{array}{c}1.3 \\
(0.8)\end{array}$ & $\begin{array}{c}0.6 \\
(0.4)\end{array}$ & $\begin{array}{c}1.0 \\
(0.4)\end{array}$ & $\begin{array}{c}0.9 \\
(0.3)\end{array}$ \\
\hline
\end{tabular}

c

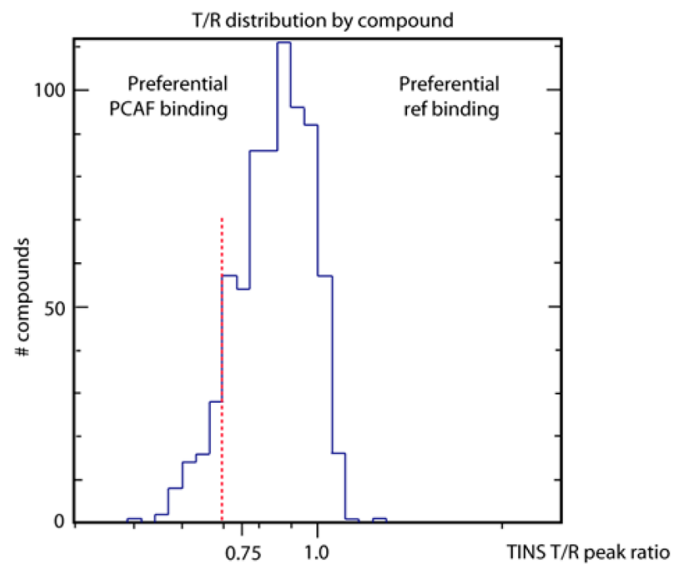

Figure 2. Fragments containing Kac mimetics identified as potential binders for PCAF bromodomain. Chemical structures of the identified fragments are shown in (a) together with average $T_{\mathrm{m}}$ shifts $( \pm$ SEM) using triplicate experimental data listed in (b). (c) TINS profile. The $T / R$ ratio of each compound screened was calculated as a height weighted average of the ratio of the peak amplitude of each NMR resonance in the presence of PCAF over that in the presence of the reference protein. The $T / R$ ratios were then binned, and the frequency is plotted above. The asymmetry and tailing to the left (i.e., a large number of compounds displaying preferential binding to PCAF) are indicative of high ligandability of PCAF. The vertical red-dashed line indicates the cutoff for definition of hits. Data of TINS hits are compiled in Supporting Information, Table S1.

available fragments. ${ }^{24}$ We first confirmed that binding of Kac to immobilized PCAF could be observed in 2\% DMSO (Supporting Information, Figure S1). Subsequently mixtures of fragments were screened with a maximum of four compounds per mix such that the DMSO concentration was never more than $2 \%$. Consistent with earlier predictions, we observed a TINS profile indicating that PCAF is highly ligandable (Figure 2c). On the basis of the profile, 63 hits were selected, a $6.6 \%$ hit rate. In contrast to TINS, binding of Kac to immobilized PCAF in the presence of DMSO could not be detected by SPR. Consequently, only those hits that were readily soluble in an aqueous buffer were orthogonally validated by SPR. The binding of 17 hits could be fully characterized by SPR, and three were selected for structural studies based on novel features (Supporting Information, Figure S2 and Table S1). These fragments $(\mathbf{9}, \mathbf{1 0}$, and 11) also resulted in substantial $T_{\mathrm{m}}$ shifts (Figure 2). 
We next attempted to verify the binding modes of the identified fragments and successfully determined the complex crystal structures for seven Kac mimetic fragments. As expected from their chemical structure, all of these fragments occupied the Kac binding site through groups that mimicked the hydrogen bond interaction of acetyl-lysine. Because the cocrystallized compounds were small, the contacts with the bromodomain were limited only to the canonical hydrogen bond with $\mathrm{N} 803$ and the typical water-mediated contact with Y760 (Figure 3a). However, additional interactions were also observed for Fr11, of which the 1-ethanol decoration was oriented toward the open ZA cavity and formed both direct and water-mediated hydrogen bonds to the backbones of the $\mathrm{ZA}$ loop V752 and P751. Superimposition of all structures revealed that the co-crystallized fragments fit tightly into the narrow Kac pocket, and most fragments formed aromatic interactions with Y809 that lines the central acetyl-lysine binding groove of the PCAF bromomdomain (Figure $3 \mathrm{~b}$ ). No significant structural alterations were observed when comparing all complexes, suggesting that the PCAF bromodomain contains a rigid acetyllysine binding pocket. Some Kac mimetic groups of the identified fragments were not specific for PCAF and have been previously shown to bind to other bromodomains, for example, 1 also interacts with ATAD $2^{11}$ and the isoxazole 8 with BRD4 and $\mathrm{CREBBP}^{25}$ with highly conserved binding modes.

With the success in identifying the Kac mimetic fragments and obtaining the complex structures, we next expanded the ligand series to more decorated compounds by choosing two diverse scaffolds (Figure 4a). Two compounds, CPD1 (12) (N(1,4-dimethyl-2-oxo-1,2-dihydroquinolin-7-yl)acetamide) and CPD2 (13) ( $N$-(1,4-dimethyl-2-oxo-1,2-dihydroquinolin-7-yl)methanesulfonam), were derivatives based on fragment hit 1 containing an additional 4-methyl group and two different decorations at the seventh position, either with N-linked acetamide or sulfonamide. A third inhibitor, CPD3 (14) $(\mathrm{N}$ methyl-5-(2-oxo-2-(phenylamino)ethoxy)-2-((tetrahydro- $2 \mathrm{H}$ pyran-4-yl)oxy)benzamide), contained instead a benzene core harboring an acetamide extension, a Kac mimetic group resembling fragment $\mathbf{1}$. In addition, this latter compound was also further decorated by tetrahydropyran and $\mathrm{N}$-phenylacetamide linked to the benzene ring via an ethoxy linkage at the ortho and meta positions relative to the acetamide group, respectively.

The binding of these three compounds was initially confirmed by DSF with 12 and 13, showing a similar degree of stabilization with $\Delta T_{\mathrm{m}}$ shifts of $\sim 2.6-2.9{ }^{\circ} \mathrm{C}$ at $1 \mathrm{mM}$ concentration (Figure 4b). Because of signal interference, 14 was used at half the concentration of the others but nonetheless produced a similar $\Delta T_{\mathrm{m}}$ shift of $\sim 3.3{ }^{\circ} \mathrm{C}$. As expected, the increase of the $\Delta T_{\mathrm{m}}$ shift values of these three ligands suggested stronger binding to PCAF bromodomain. We used isothermal titration calorimetry (ITC) to determine binding affinities in solution.

In agreement with the DSF results, the measured $K_{\mathrm{d}}$ of $\mathbf{1 3}$ was observed to be $\sim 21 \mu \mathrm{M}$, which was 3-fold higher than that of the more decorated $14\left(K_{d}\right.$ of $\left.\sim 7 \mu \mathrm{M}\right)$ (Figure $\left.4 \mathrm{c}\right)$. The increase in the affinity of the latter was due to significantly larger negative binding enthalpy, suggesting more favorable polar interaction of this inhibitor. However, the ligand efficiency (LE) decreased to 0.24 for 14 compared to LE of 0.34 for 13. To gain insight into the selectivity of the most potent fragment hits, we performed a temperature shift screen using a panel of 48 recombinant bromodomains (Supporting
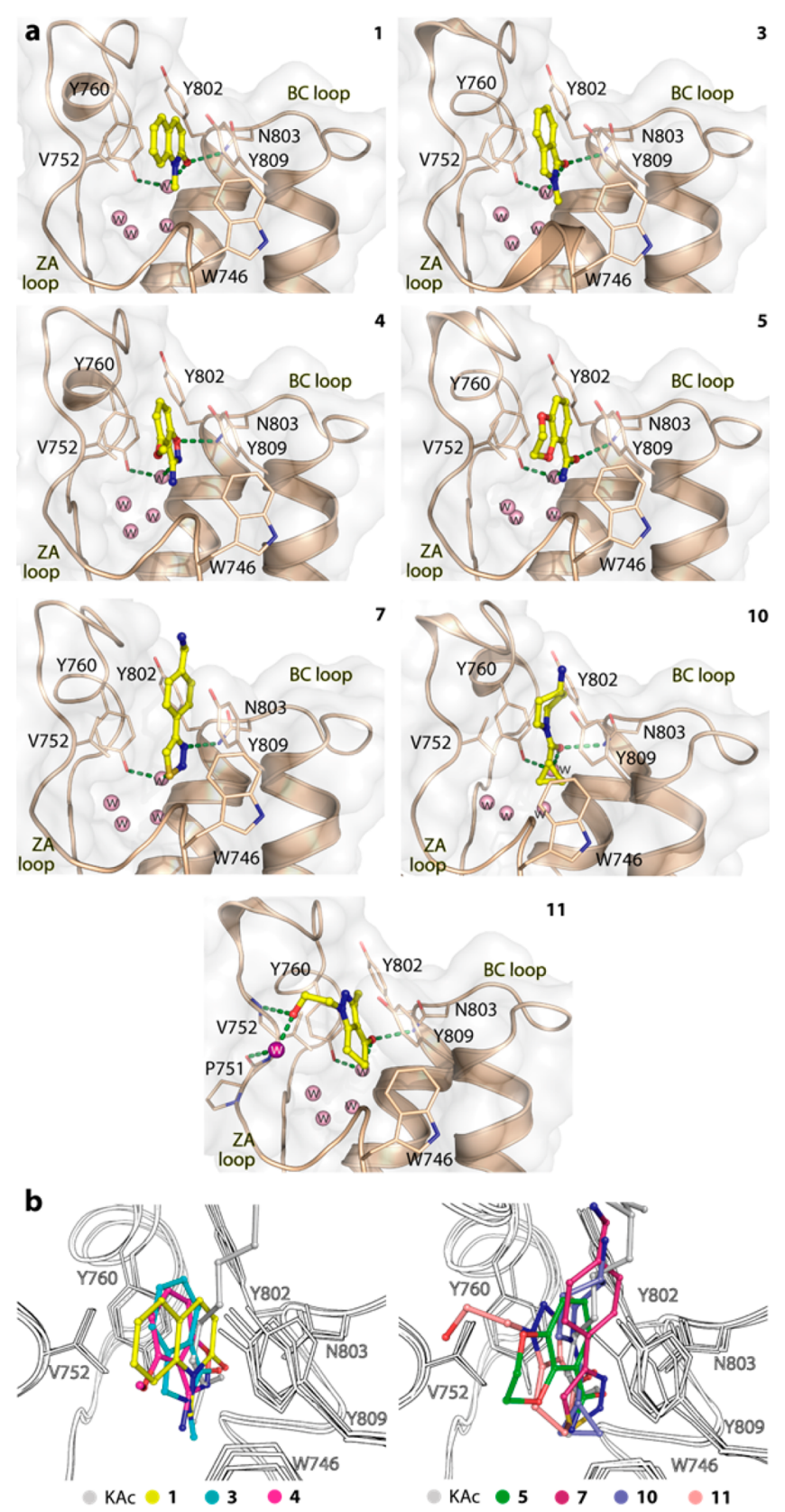

Figure 3. Structures of PCAF in complexes with the identified Kac mimetic fragments. (a) Detailed interactions between the bound fragments (yellow stick) within the PCAF Kac binding site. The conserved water molecules at the bottom of the pocket are shown in pink spheres, and an additional water molecule involving in additional water-mediated interactions observed in the complex with 11 is highlighted by magenta sphere. (b) Superimposition of the bound fragments and Kac revealed canonical acetyl-lysine mimetic binding modes and no significant conformational changes upon inhibitor binding within the pocket. Coordinates have been deposited under accession codes: 5fe1, 5fe2, 5fe3, 5fe4, 5fe6, 5fe7, and 5fe5.

Information, Figure S3). This selectivity screen revealed strong interaction of 14 to bromodomains present in CECR2 (7.1 $\left.{ }^{\circ} \mathrm{C}\right)$, BRD7 $\left(6.5^{\circ} \mathrm{C}\right)$, suggesting that this fragment can also be optimized targeting other bromodomains. 12 and 13 were also screened using developed Alphascreen assays. At $25 \mu \mathrm{M}$, both inhibitors showed strong inhibition for BRD9 (Supporting Information, Table S2). This is not surprising because 1- 
<smiles>CC(=O)Nc1ccc2c(C)cc(=O)n(C)c2c1</smiles><smiles>CNC(=O)c1cc(OCC(=O)Nc2ccccc2)ccc1OC1CCOCC1</smiles>

b

\begin{tabular}{c|c|}
\hline $\begin{array}{r}\text { Compounds } \\
\text { (concentration) }\end{array}$ & $\begin{array}{r}\Delta \mathrm{Tm}\left({ }^{\circ} \mathrm{C}\right) \\
( \pm \mathrm{SEM})\end{array}$ \\
\hline $\mathbf{1 2}(1 \mathrm{mM})$ & $2.6(0.3)$ \\
\hline $\mathbf{1 3}(1 \mathrm{mM})$ & $2.9(0.5)$ \\
\hline $\mathbf{1 4}(0.5 \mathrm{mM})$ & $3.3(0.1)$ \\
\hline
\end{tabular}

C 13
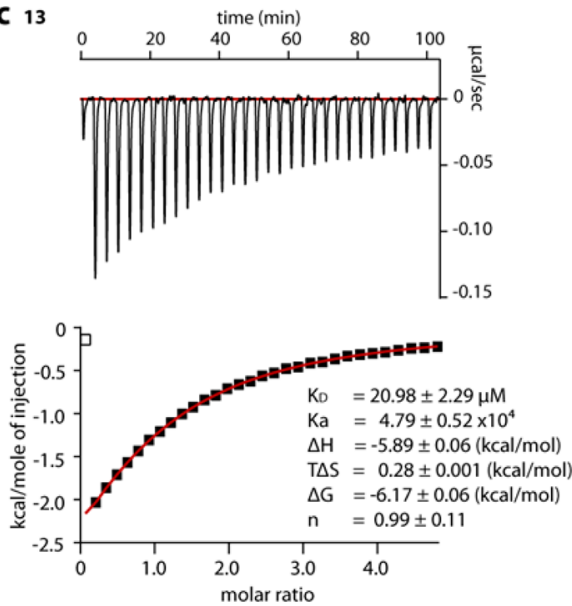
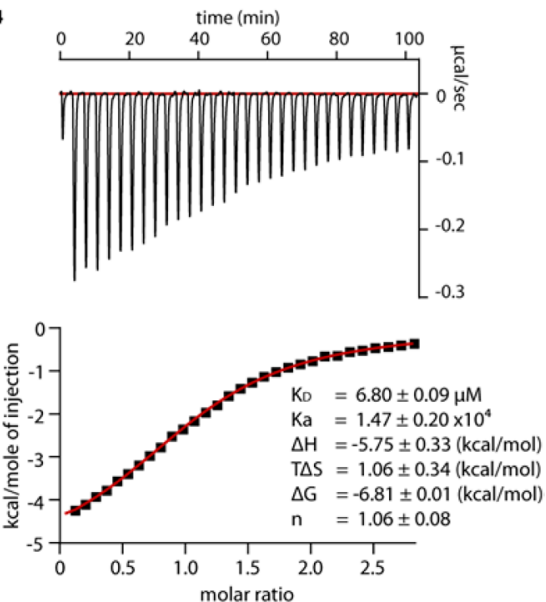

Figure 4. Compounds identified as PCAF binders. (a) Chemical structures of three in-house available compounds that contain the fragment-related Kac mimetic scaffolds. (b) Average $T_{\mathrm{m}}$ shift values from triplicate experiments for the selected compounds. (c) ITC binding data for the interactions between 13 and 14 with PCAF with the isotherms of raw titration heat shown on the top and the normalized binding heat with the single-site fitting (red line) shown at the bottom. The thermodynamic parameters including equilibrium binding constants are the average from two repeats.

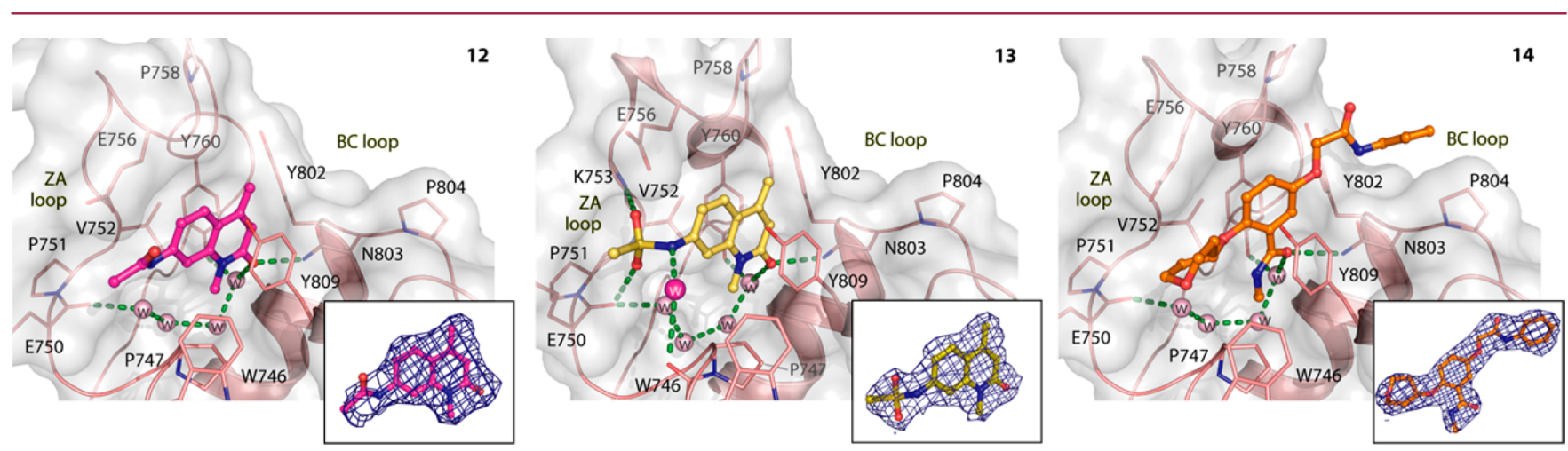

Figure 5. Crystal structures of PCAF in complexes with 12, 13, and 14. Detailed interactions between the bound compounds with the PCAF bromodomain are shown. The conserved water at the bottom of the pocket is displayed in pink spheres, while an additional water molecule involving in contact between the $\mathbf{1 3}$ and protein is shown in magenta sphere. Insets show omitted electron density map for the bound ligands. Coordinates have been deposited under accession codes: $5 \mathrm{fe} 8,5 \mathrm{fe} 9$, and $5 \mathrm{fdz}$, respectively.

methylquinoline-2-ones have been developed into highly specific BRD9 inhibitors. ${ }^{10}$

The crystal structures of the ligand-PCAF complex allowed us to evaluate the interactions of the introduced decorations. All compounds could be unambiguously positioned based on the well-defined electron density map (Figure 5). As expected in complexes with 12 and 13, the 1-methylquinoline-2-one group occupied the Kac binding groove and formed similar canonical interactions as observed in the PCAF complex with $\mathbf{1}$, while the decoration of both compounds oriented toward the ZA pockets. However, no contact between the 12 acetamide and protein was observed. In contrast, the $13 \mathrm{~N}$-linked sulfonamide was observed to replace a water molecule that bridged interactions between E756 and K753 in the Kac complex (Figure 1a), enabling the formation of two direct hydrogen bonds to these two residues as well as a watermediated contact to the carbonyl backbone of W746 (Figure 5). Interestingly, a sulfonamide at this analogous position also engaged within the Kac site of BAZ2B in the GSK2801 inhibitor complex. ${ }^{5}$

The binding mode of $\mathbf{1 4}$ was slightly different potentially due to its larger decorations and its different acetyl-lysine mimetic moiety (Figure 5). The acetamide interacted with PCAF in a typical Kac mimetic manner, with the benzene core stacking against the Y809 in a similar fashion observed also for the other fragments. The tetrahydropyran expanded into the open ZA pocket, while the $\mathrm{N}$-phenylacetamide extension occupied the BC pocket with the phenyl ring located above N803 and oriented perpendicularly to Y802 and P804 within a distance for $\pi$-stacking. However, no hydrogen bond contact was formed between these substitutions and PCAF. Considering its affinity, such limited interactions were rather surprising when compared to the binding mode of the weaker 13 , of which the extended group engaged in more hydrogen bond interactions. We hypothesize therefore that $\mathbf{1 4}$ may gain its increased binding potency through a larger contact surface. Hence, an extension of the Kac-mimetic fragments resulted in improved binding affinities despite the lack of direct polar interaction with the protein. In addition, the large surrounding space adjacent to the ZA and BC loops provides few spatial constraints, enabling the binding of diverse chemical moieties, albeit with little shape complementarity. It is likely that elongated, flexible, and uncharged substituents that could span the interface as well as engage a few interactions may be beneficial for increasing ligand potency through targeting the $\mathrm{ZA}$ and $\mathrm{BC}$ pockets in the PCAF bromodomain. 


\section{CONCLUSION}

The fragment-based approach coupled with structural information presented here offers a platform expanding the knowledge on PCAF Kac-mimetic scaffolds and binding modes to aid development of potent and selective PCAF bromodomain inhibitors. The structural models provide valuable data for the rational design of inhibitors to the rigid and poorly enclosed PCAF Kac binding pocket. Comparison of inhibitor binding modes highlights the importance of aromatic interactions with Y809 and provides several diverse acetyl-lysine mimetic chemotypes. These chemotypes may serve as attractive starting points for inhibitor development efforts targeting this interesting bromodomain. All fragments that were cocrystallized formed an acetyl-lysine mimetic hydrogen bond to N803. Thus, for future development of these fragments into more potent hits the fragment need to be grown rather than linked in order to increase potency.

\section{EXPERIMENTAL SECTION}

Recombinant Protein Production. The PCAF bromodomain (aa 715-831) containing an $\mathrm{N}$-terminal $\mathrm{His}_{6}$-tag was recombinantly expressed in Escherichia coli Rosetta. The protein was initially purified by $\mathrm{Ni}^{2+}$-affinity chromatography and subsequently cleaved the tag with TEV protease. The cleaved protein was further purified using size exclusion chromatography and stored in $25 \mathrm{mM}$ HEPES, $\mathrm{pH}$ 7.5, 150 $\mathrm{mM} \mathrm{NaCl}$, and $0.5 \mathrm{mM}$ TCEP.

Identified Fragments and Compounds. Fragments were purchased from chemical vendors and were at least $95 \%$ pure according vendor specifications. The purity and correct structure of each compound was validated by NMR. The purity of the synthesized compounds 12, 13, and 14 was assessed by analytical HPLC using an Agilent 1100 equipped with photodiode array detector (DAD), quaternary gradient pump, and micro plate sampler (Agilent 220). Separation was performed upon Centurysil C18-AQ $+5 \mu \mathrm{m}, 50 \mathrm{~mm} \times$ $4.6 \mathrm{~mm}$ (Johnson). The flow rate of the mobile phase was kept at 3.5 $\mathrm{mL} / \mathrm{min}$. Mobile phases $\mathrm{B}$ and $\mathrm{C}$ were acetonitrile with $0.35 \%$ $\mathrm{CF}_{3} \mathrm{CO}_{2} \mathrm{H}$ and water with $0.35 \% \mathrm{CF}_{3} \mathrm{CO}_{2} \mathrm{H}$, respectively. The gradient conditions were as follows: $0-0.5 \mathrm{~min} 1 \% \mathrm{~B}$ and $99 \% \mathrm{C}, 3.7 \mathrm{~min} 90 \%$ $\mathrm{B}$ and $10 \%$ C, 5 min $99 \% \mathrm{~B}$ and $1 \%$ C. The injection volume was 10 $\mu \mathrm{L} .12$ and 13 were $\geq 90 \%$ pure by HPLC at $254 \mathrm{~nm}$ and $\geq 99 \%$ by evaporative light scattering detection (ELSD). 14 was $\geq 99 \%$ pure by both methods.

Thermal Stability Assays. The protein at $2 \mu \mathrm{M}$ in $10 \mathrm{mM}$ HEPES, pH 7.5 and $250 \mathrm{mM} \mathrm{NaCl}$ was mixed with the fragments/ compounds at $1 \mathrm{mM}$ concentration (unless stated). The assays, data evaluation, and melting temperature $\left(T_{\mathrm{m}}\right)$ calculation were performed using a Real-Time PCR Mx3005p machine (Stratagene) with the protocols described previously. ${ }^{26}$

Isothermal Calorimetry. All isothermal calorimetry (ITC) experiments were carried out on NanoITC (TA Instruments) at 15 ${ }^{\circ} \mathrm{C}$ in the buffer containing $25 \mathrm{mM}$ HEPES, $\mathrm{pH} 7.5,150 \mathrm{mM} \mathrm{NaCl}$, and $0.5 \mathrm{mM}$ TCEP. Titration was performed by injecting PCAF (250 $\mu \mathrm{M})$ into the reaction cell containing the compounds $(15-20 \mu \mathrm{M})$. The corrected data of the integrated heat of titration were fitted to an independent single binding site model based on the manufactor protocol, from which the thermodynamics parameters $(\Delta H$ and $T \Delta S)$, equilibrium association and dissociation constants $\left(K_{\mathrm{a}}\right.$ and $\left.K_{\mathrm{D}}\right)$, and stoichiometry $(n)$ were calculated. All experiments were repeated twice.

SPR. Recombinant PCAF was biotinylated on the N-terminal AVI tag in vivo. Purified, biotinylated PCAF was captured onto a NeutraAvidin-coated CM5 sensor chip surface in $20 \mathrm{mM}$ sodium phosphate pH7.5, $150 \mathrm{mM} \mathrm{NaCl}, 2 \mathrm{mM}$ DTT, and 0.05\% P20 Tween. This buffer was subsequently used for all studies. The immobilization level was 10150 RU. All SPR experiments were performed using a Biacore T200 instrument at $20{ }^{\circ} \mathrm{C}$. Fragment hits from TINS were directly solubilized in the running buffer. Each fragment was titrated at six different concentrations for $K_{\mathrm{D}}$ determination. An acetyl-Lys 16 peptide derived from $\mathrm{H} 4$ was used to monitor the ligand binding capacity of the immobilized PCAF bromodomain at regular intervals.

Crystallization and Structure Determination. Apo crystals of PCAF $(\sim 14-18 \mathrm{mg} / \mathrm{mL})$ were obtained using the vapor diffusion method at $4{ }^{\circ} \mathrm{C}$ and either single-PEG-based (21-35\% PEG 3350, 0.1 $\mathrm{M}$ Bis-Tris pH 5.5-7.0) or PEG-smear-based (21-40\% mediummolecular-weight PEG smears buffered either with $0.1 \mathrm{M}$ Bis-Tris $\mathrm{pH}$ $6.0-7.5$ or $0.1 \mathrm{M}$ Tris $\mathrm{pH} 7.5-8.8)^{27}$ reservoir solutions. To produce the PCAF-ligand complex crystals, soaking was performed using the mother liquor supplemented with the fragments/compounds at $\sim 10-$ $20 \mathrm{mM}$ concentration and $20 \%$ ethylene glycol. Diffraction data were collected either in-house or at the Diamond Light Source. Initial structure solution was achieved using molecular replacement with Phaser $^{28}$ from CCP4 suite ${ }^{29}$ and the published coordinates of PCAF. ${ }^{23}$ Manual model building alternated with refinement were performed in $\mathrm{COOT}^{30}$ and Refmac, ${ }^{31}$ respectively. Data collection and refinement statistics are summarized in Supporting Information, Table S3.

\section{ASSOCIATED CONTENT}

\section{S Supporting Information}

The Supporting Information is available free of charge on the ACS Publications website at DOI: 10.1021/acs.jmedchem.5b01719.

Crystallographic data collection and refinement statistics, compound syntheses, selectivity screening data (PDF)

\section{Accession Codes}

The coordinates and structure factors of all complexes have been deposited to the protein data bank under accession codes $5 \mathrm{fdz}, 5 \mathrm{fe} 0$, 5fe1, 5fe2, 5fe3, 5fe4, 5fe5, 5fe6, 5fe 7, 5fe8, and $5 \mathrm{fe} 9$.

\section{AUTHOR INFORMATION}

\section{Corresponding Author}

*Phone: +49 69798 12799. E-mail: knapp@pharmchem.unifrankfurt.de. Address: Goethe University Frankfurt, Department of Pharmaceutical Chemistry and Buchmann Institute for Molecular Life Sciences, 60438 Frankfurt, Germany.

\section{Author Contributions}

All authors approved the final manuscript.

\section{Notes}

The authors declare no competing financial interest.

\section{ACKNOWLEDGMENTS}

We thank the staff at Diamond Light Source for assistance during data collection. The SGC is a registered charity (no. 1097737) that receives funds from AbbVie, Bayer, Boehringer Ingelheim, the Canada Foundation for Innovation, the Canadian Institutes for Health Research, Genome Canada, GlaxoSmithKline, Janssen, Lilly Canada, the Novartis Research Foundation, the Ontario Ministry of Economic Development and Innovation, Pfizer, Takeda, and the Wellcome Trust $[092809 / \mathrm{Z} / 10 / \mathrm{Z}]$. A.C. has been supported by the European Union FP7 grant no. 278568 "PRIMES". S.L. was supported by a German Academic Exchange Programme DAAD Postdoctoral Fellowship.

\section{ABBREVIATIONS USED}

PCAF, p300/CBP-associated factor; Kac, $N \varepsilon$-acetyllysine; HIV, human immunodeficiency virus; AIDS, acquired immune deficiency syndrome; ATAD2, ATPase family AAA domaincontaining protein 2 ; BAZ2B, bromodomain adjacent to zinc finger domain protein $2 \mathrm{~B} ; \mathrm{BET}$, bromodomain and extraterminal; BRD4, bromodomain-containing protein 4; CREBBP, 
CREB-binding protein; SPR, surface plasmon resonance; TINS, target immobilized NMR screening

\section{REFERENCES}

(1) Filippakopoulos, P.; Knapp, S. Targeting bromodomains: epigenetic readers of lysine acetylation. Nat. Rev. Drug Discovery 2014, 13, 337-356.

(2) Muller, S.; Filippakopoulos, P.; Knapp, S. Bromodomains as therapeutic targets. Expert Rev. Mol. Med. 2011, 13, e29.

(3) Vidler, L. R.; Brown, N.; Knapp, S.; Hoelder, S. Druggability analysis and structural classification of bromodomain acetyl-lysine binding sites. J. Med. Chem. 2012, 55, 7346-7359.

(4) Drouin, L.; McGrath, S.; Vidler, L. R.; Chaikuad, A.; Monteiro, O.; Tallant, C.; Philpott, M.; Rogers, C.; Fedorov, O.; Liu, M.; Akhtar, W.; Hayes, A.; Raynaud, F.; Muller, S.; Knapp, S.; Hoelder, S. Structure enabled design of BAZ2-ICR, a chemical probe targeting the bromodomains of BAZ2A and BAZ2B. J. Med. Chem. 2015, 58, 25532559.

(5) Chen, P.; Chaikuad, A.; Bamborough, P.; Bantscheff, M.; Bountra, C.; Chung, C. W.; Fedorov, O.; Grandi, P.; Jung, D.; Lesniak, R.; Lindon, M.; Muller, S.; Philpott, M.; Prinjha, R.; Rogers, C.; Selenski, C.; Tallant, C.; Werner, T.; Willson, T. M.; Knapp, S.; Drewry, D. H. Discovery and Characterization of GSK2801, a Selective Chemical Probe for the Bromodomains BAZ2A and BAZ2B. J. Med. Chem. 2016, DOI: 10.1021/acs.jmedchem.5b00209.

(6) Bamborough, P.; Chung, C. W.; Furze, R. C.; Grandi, P.; Michon, A. M.; Sheppard, R. J.; Barnett, H.; Diallo, H.; Dixon, D. P.; Douault, C.; Jones, E. J.; Karamshi, B.; Mitchell, D. J.; Prinjha, R. K.; Rau, C.; Watson, R. J.; Werner, T.; Demont, E. H. Structure-Based Optimization of Naphthyridones into Potent ATAD2 Bromodomain Inhibitors. J. Med. Chem. 2015, 58, 6151.

(7) Bennett, J.; Fedorov, O.; Tallant, C.; Monteiro, O.; Meier, J.; Gamble, V.; Savitsky, P.; Nunez-Alonso, G. A.; Haendler, B.; Rogers, C.; Brennan, P. E.; Muller, S.; Knapp, S. Discovery of a Chemical Tool Inhibitor Targeting the Bromodomains of TRIM24 and BRPF. J. Med. Chem. 201610.1021/acs.jmedchem.5b00458.

(8) Demont, E. H.; Chung, C. W.; Furze, R. C.; Grandi, P.; Michon, A. M.; Wellaway, C.; Barrett, N.; Bridges, A. M.; Craggs, P. D.; Diallo, H.; Dixon, D. P.; Douault, C.; Emmons, A. J.; Jones, E. J.; Karamshi, B. V.; Locke, K.; Mitchell, D. J.; Mouzon, B. H.; Prinjha, R. K.; Roberts, A. D.; Sheppard, R. J.; Watson, R. J.; Bamborough, P. Fragment-Based Discovery of Low-Micromolar ATAD2 Bromodomain Inhibitors. J. Med. Chem. 2015, 58, 5649-5673.

(9) Hay, D. A.; Fedorov, O.; Martin, S.; Singleton, D. C.; Tallant, C.; Wells, C.; Picaud, S.; Philpott, M.; Monteiro, O. P.; Rogers, C. M.; Conway, S. J.; Rooney, T. P.; Tumber, A.; Yapp, C.; Filippakopoulos, P.; Bunnage, M. E.; Muller, S.; Knapp, S.; Schofield, C. J.; Brennan, P. E. Discovery and Optimization of Small-Molecule Ligands for the CBP/p300 Bromodomains. J. Am. Chem. Soc. 2014, 136, 9308-9319. (10) Clark, P. G.; Vieira, L. C.; Tallant, C.; Fedorov, O.; Singleton, D. C.; Rogers, C. M.; Monteiro, O. P.; Bennett, J. M.; Baronio, R.; Muller, S.; Daniels, D. L.; Mendez, J.; Knapp, S.; Brennan, P. E.; Dixon, D. J. LP99: Discovery and Synthesis of the First Selective BRD7/9 Bromodomain Inhibitor. Angew. Chem., Int. Ed. 2015, 54, 6217-6221.

(11) Chaikuad, A.; Petros, A. M.; Fedorov, O.; Xu, J.; Knapp, S. Structure-based approaches towards identification of fragments for the low-druggability ATAD2 bromodomain. MedChemComm 2014, 5, 1843-1848.

(12) Ferguson, F. M.; Fedorov, O.; Chaikuad, A.; Philpott, M.; Muniz, J. R.; Felletar, I.; von Delft, F.; Heightman, T.; Knapp, S.; Abell, C.; Ciulli, A. Targeting low-druggability bromodomains: fragment based screening and inhibitor design against the BAZ2B bromodomain. J. Med. Chem. 2013, 56, 10183-10187.

(13) Bannister, A. J.; Kouzarides, T. The CBP co-activator is a histone acetyltransferase. Nature 1996, 384, 641-643.

(14) Ogryzko, V. V.; Schiltz, R. L.; Russanova, V.; Howard, B. H.; Nakatani, Y. The transcriptional coactivators p300 and CBP are histone acetyltransferases. Cell 1996, 87, 953-959.
(15) Yang, X. J.; Ogryzko, V. V.; Nishikawa, J.; Howard, B. H.; Nakatani, Y. A p300/CBP-associated factor that competes with the adenoviral oncoprotein E1A. Nature 1996, 382, 319-324.

(16) Linares, L. K.; Kiernan, R.; Triboulet, R.; Chable-Bessia, C.; Latreille, D.; Cuvier, O.; Lacroix, M.; Le Cam, L.; Coux, O.; Benkirane, M. Intrinsic ubiquitination activity of PCAF controls the stability of the oncoprotein Hdm2. Nat. Cell Biol. 2007, 9, 331-338.

(17) Lau, O. D.; Courtney, A. D.; Vassilev, A.; Marzilli, L. A.; Cotter, R. J.; Nakatani, Y.; Cole, P. A. p300/CBP-associated factor histone acetyltransferase processing of a peptide substrate. Kinetic analysis of the catalytic mechanism. J. Biol. Chem. 2000, 275, 21953-21959.

(18) Lau, O. D.; Kundu, T. K.; Soccio, R. E.; Ait-Si-Ali, S.; Khalil, E. M.; Vassilev, A.; Wolffe, A. P.; Nakatani, Y.; Roeder, R. G.; Cole, P. A. HATs off: selective synthetic inhibitors of the histone acetyltransferases p300 and PCAF. Mol. Cell 2000, 5, 589-595.

(19) Mujtaba, S.; He, Y.; Zeng, L.; Farooq, A.; Carlson, J. E.; Ott, M.; Verdin, E.; Zhou, M. M. Structural basis of lysine-acetylated HIV-1 Tat recognition by PCAF bromodomain. Mol. Cell 2002, 9, 575-586.

(20) Dorr, A.; Kiermer, V.; Pedal, A.; Rackwitz, H. R.; Henklein, P.; Schubert, U.; Zhou, M. M.; Verdin, E.; Ott, M. Transcriptional synergy between Tat and PCAF is dependent on the binding of acetylated Tat to the PCAF bromodomain. EMBO J. 2002, 21, 2715-2723.

(21) Zeng, L.; Li, J.; Muller, M.; Yan, S.; Mujtaba, S.; Pan, C.; Wang, Z.; Zhou, M. M. Selective small molecules blocking HIV-1 Tat and coactivator PCAF association. J. Am. Chem. Soc. 2005, 127, 23762377.

(22) Hu, P.; Wang, X.; Zhang, B.; Zhang, S.; Wang, Q.; Wang, Z. Fluorescence polarization for the evaluation of small-molecule inhibitors of PCAF BRD/Tat-AcK50 association. ChemMedChem 2014, 9, 928-931.

(23) Filippakopoulos, P.; Picaud, S.; Mangos, M.; Keates, T.; Lambert, J. P.; Barsyte-Lovejoy, D.; Felletar, I.; Volkmer, R.; Muller, S.; Pawson, T.; Gingras, A. C.; Arrowsmith, C. H.; Knapp, S. Histone recognition and large-scale structural analysis of the human bromodomain family. Cell 2012, 149, 214-231.

(24) Vanwetswinkel, S.; Heetebrij, R. J.; van Duynhoven, J.; Hollander, J. G.; Filippov, D. V.; Hajduk, P. J.; Siegal, G. TINS, target immobilized NMR screening: an efficient and sensitive method for ligand discovery. Chem. Biol. 2005, 12, 207-216.

(25) Hewings, D. S.; Fedorov, O.; Filippakopoulos, P.; Martin, S.; Picaud, S.; Tumber, A.; Wells, C.; Olcina, M. M.; Freeman, K.; Gill, A.; Ritchie, A. J.; Sheppard, D. W.; Russell, A. J.; Hammond, E. M.; Knapp, S.; Brennan, P. E.; Conway, S. J. Optimization of 3,5dimethylisoxazole derivatives as potent bromodomain ligands. J. Med. Chem. 2013, 56, 3217-3227.

(26) Fedorov, O.; Niesen, F. H.; Knapp, S. Kinase inhibitor selectivity profiling using differential scanning fluorimetry. Methods Mol. Biol. 2012, 795, 109-118.

(27) Chaikuad, A.; Knapp, S.; von Delft, F. Defined PEG smears as an alternative approach to enhance the search for crystallization conditions and crystal-quality improvement in reduced screens. Acta Crystallogr., Sect. D: Biol. Crystallogr. 2015, 71, 1627-1639.

(28) McCoy, A. J.; Grosse-Kunstleve, R. W.; Adams, P. D.; Winn, M. D.; Storoni, L. C.; Read, R. J. Phaser crystallographic software. J. Appl. Crystallogr. 2007, 40, 658-674.

(29) Winn, M. D.; Ballard, C. C.; Cowtan, K. D.; Dodson, E. J.; Emsley, P.; Evans, P. R.; Keegan, R. M.; Krissinel, E. B.; Leslie, A. G.; McCoy, A.; McNicholas, S. J.; Murshudov, G. N.; Pannu, N. S.; Potterton, E. A.; Powell, H. R.; Read, R. J.; Vagin, A.; Wilson, K. S. Overview of the CCP4 suite and current developments. Acta Crystallogr., Sect. D: Biol. Crystallogr. 2011, 67, 235-242.

(30) Emsley, P.; Lohkamp, B.; Scott, W. G.; Cowtan, K. Features and development of Coot. Acta Crystallogr., Sect. D: Biol. Crystallogr. 2010, $66,486-501$.

(31) Skubak, P.; Murshudov, G. N.; Pannu, N. S. Direct incorporation of experimental phase information in model refinement. Acta Crystallogr., Sect. D: Biol. Crystallogr. 2004, 60, 2196-2201. 